mation of a thick layer around the microsphere. The researchers said that both limiting tests agree reasonably with theory.

The researchers then characterized a nanolayer composed of poly-L-lysine hydrogel, which, due to its very high charge, readily adsorbs negatively charged biomolecules. Poly-L-lysine hydrogel nanolayers are also difficult to characterize using other methods because it forms such thin layers with low contrast to water. The researchers observed a very small resonance shift, which their theory indicates corresponds to a layer thickness of $110 \mathrm{~nm}$ and an excess refractive index of 0.0012 . The researchers said that as a result of their method, "the WGM resonator goes beyond its original promise as a biosensor." In addition, the researchers anticipate that real-time measurement of $S$ will reveal morphology changes concomitant with increases in layer density. Furthermore, Arnold and his co-researchers said that an alternate formulation of their theory applied to nonspherical particles "shows promise for looking at heterogeneous structures such as adsorbed bacteria."

STEVEN TROHALAKI

\section{Metalorganic Gel Used for Porous Organic Polymer Template}

Coordination polymers where metal centers are linked by organic bridging ligands represent novel materials with potentially useful porosity and inclusion properties. Much of the research on this class of materials has, however, focused on studying single crystals of these materials to understand the molecular basis for their formation and properties. Polymer gels-which potentially have interesting properties in catalysis, sensing, and as responsive materials - have been far less studied. In the March 28 issue of Chemical Communications (DOI: $10.1039 / \mathrm{b} 418554 \mathrm{~d}), \mathrm{Q}$. Wei and S.L. James from the Queen's University of Belfast in Northern Ireland report the reaction of iron nitrate and 1,3,5-benzenetricarboxylic acid in ethanol to give a metalorganic gel with a solvent content of $95-98 \%$ by weight.

The researchers propose that coordination polymer particles are formed by rapid cross-linking polymerization between $\mathrm{Fe}^{3+}$ and the benzenetricarboxylic acid and that these particles are further crosslinked to provide macropores that trap solvent molecules. The gel can be formed in the presence of organic monomers, which can be trapped in the cavities and polymerized to form porous imprints of the metalorganic gel. Wei and James demonstrate this for poly(methyl methacrylate) (PMMA) by polymerizing methyl methacrylate in the gel cavities and dissolving the gel framework in hydrochloric acid. The resulting PMMA imprint contains disordered pores with a size range of $1-10 \mu \mathrm{m}$.

James said that the major advantage of this technique is that it is a straightforward and inexpensive route to templating porous organic polymers, which have potential applications in supports and separations. The presence of metalorganic particles within the polymer matrix suggests that other interesting magnetic and responsive properties may be observable in these materials, the researchers said.

SARBAJTT BANERJEE

\section{Tungsten Nanoparticles Embedded in Silica \\ Enhance Nonvolatile Memory}

Many consumer electronic products use nonvolatile memory devices in their operation. One such device is electrically erasable and programmable read-only memory (EEPROM), which uses a floating gate structure. In the drive to develop memories with lower power consumption and faster erase/write cycles, researchers have begun to incorporate nanocrystals into their EEPROMs in order to improve performance. Recently, T.C. Chang of the National Sun Yat-Sen University, P.T. Liu of the National Chiao Tung University, and their colleagues demonstrated that replacing the floating gate in EEPROMs with tungsten nanocrystals in a silica matrix reduces the operating voltage and increases endurance. Using nanoparticles in
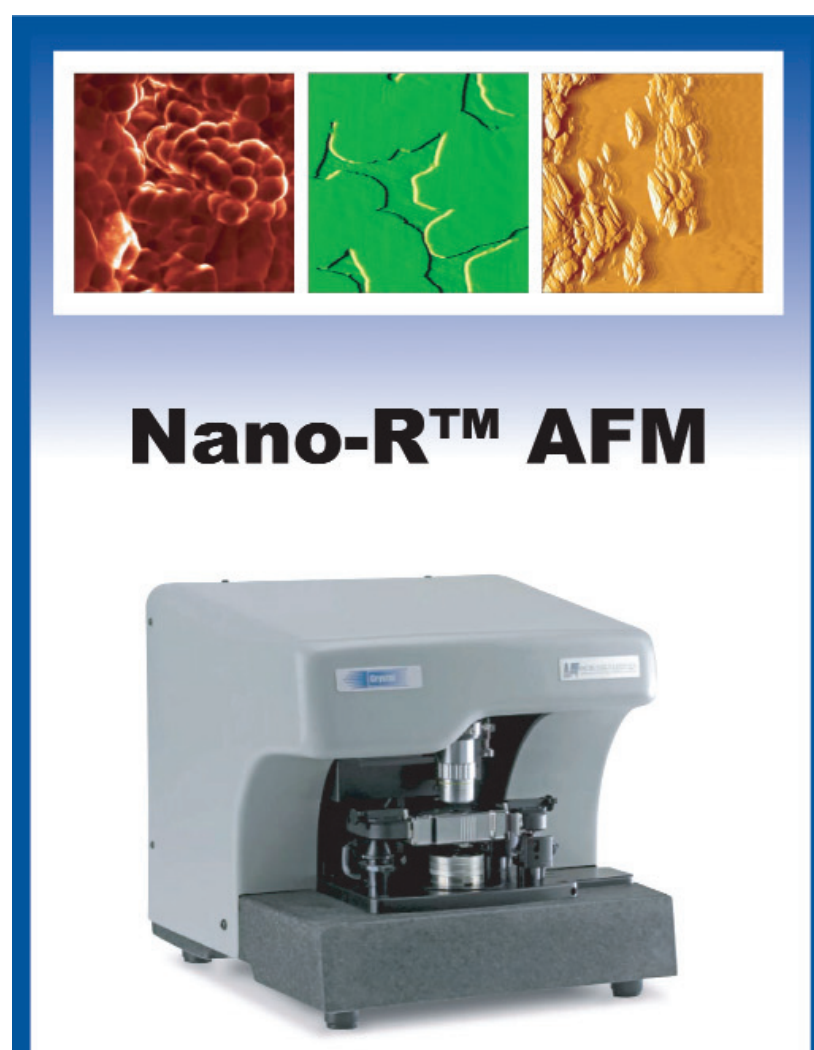

- High Performance

- Easy to Use

- Versatile

Nano- $R^{\mathrm{TM}}$ is available with a traditional light lever scanner and the advanced Crystal Scanner ${ }^{\mathrm{TM}}$.

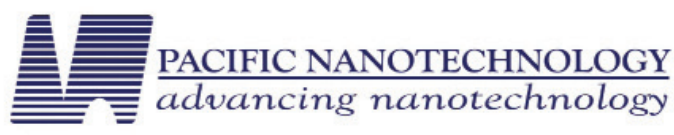

3350 Scott Blvd. \#29

Santa Clara, CA 95054

408-982-9492

www.pacificnanotech.com

www.probestore.com
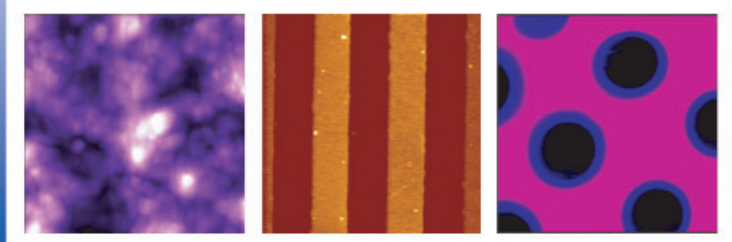

For more information, see http://advertisers.mrs.org 
the floating gate makes the structure more tolerant to defects in the tunnel oxide and therefore reduces leakage. This permits the use of thinner tunnel oxides, which in turn reduces the operating voltage, improves endurance and retention, and increases the write/erase rate.

To fabricate their devices, the researchers took advantage of the fact that tungsten silicide on $\mathrm{SiO}_{2}$ forms elemental tungsten when thermally oxidized. As described in the March issue of Electrochemical and SolidState Letters (p. G71), the team began by growing a $4.5 \mathrm{~nm}$ tunnel oxide on silicon substrates, followed by sputter deposition of $8 \mathrm{~nm}$ of $\mathrm{W}_{5} \mathrm{Si}_{3}$ for the floating gate and 10 $\mathrm{nm}$ of amorphous silicon. A thermal oxidation step formed the control oxide and the elemental $\mathrm{W}$ in the $\mathrm{SiO}_{2}$ matrix. The $\mathrm{W}$ particles had an average diameter of $4.5 \mathrm{~nm}$ and an areal density of $3.7 \times 10^{11} \mathrm{~cm}^{-2}$. Fourier transform infrared spectroscopy analysis showed that elemental tungsten was clearly present, with little or no tungsten oxide formation.

The researchers used capacitance-voltage measurements to demonstrate the electron charging effect of their $\mathrm{W}$ particles. A bidirectional voltage sweep from $3 \mathrm{~V}$ to $-4 \mathrm{~V}$ and back to $3 \mathrm{~V}$ yielded a shift in the threshold voltage $\Delta V_{\mathrm{t}}$ of $0.95 \mathrm{~V}$, which is sufficient for binary switching. The researchers found that there was no appreciable decrease in $\Delta V_{\mathrm{t}}$ until after $10^{6}$ cycles and in fact, after $10^{9}$ cycles, $\Delta V_{\mathrm{t}}$ had only dropped to $0.71 \mathrm{~V}$.

The operating characteristics of the $\mathrm{W}$ nanoparticle EEPROMs represent an improvement over both the high $7 \mathrm{~V}$ operating voltage of conventional floating gate devices and the rapid drop-off seen in the endurance of other nanoparticle devices. In addition, this silicide technique is easily integrated with existing semiconductor technology, making the W nanocrystal devices viable competitors to conventional EEPROMs.

AMANDA GIERMANN

\section{Transparent Hydroxyapatite with High Crystal Orientation Produced by Pulsed Electric Current Sintering}

Hydroxyapatite $\left[\mathrm{Ca}_{10}\left(\mathrm{PO}_{4}\right)_{6}(\mathrm{OH}), \mathrm{HAp}\right]$ is a major mineral component found in bones and teeth. As a biomaterial, it aids the growth and development of bones and teeth by providing attachment sites for new cells. HAp has a hexagonal crystal structure and there is a large difference in cell attachment between the $a$ plane and the $c$ plane. Sintered dense HAp bodies with a high degree of crystal orientation are useful for culturing cells and as scaffolds for the regeneration of bones and teeth.
In the January issue of the Journal of American Ceramic Society (p. 243; DOI: 10.1111/j.1551-2916.2004.00041.x), researchers Yujiro Watanabe of Hosei University, Japan, and Toshiyuki Ikoma of the National Institute for Materials Science, Japan, and colleagues reported a sintering process for producing dense, transparent, and highly oriented crystalline HAp bodies.

The researchers first prepared the HAp powder by reacting $\mathrm{Ca}(\mathrm{OH})_{2}$ with $\mathrm{H}_{3} \mathrm{PO}_{4}$ solution, followed by spray drying and then calcining at $800^{\circ} \mathrm{C}$ for $3 \mathrm{~h}$. Sintering of the HAp sample was carried out by pulsed electric current sintering (PECS) in a spark plasma sintering (SPS) system. In the PECS process, powder particles are charged with electrical energy and a high pressure is applied on the sample.

During the sintering of HAp, the sample was pressed uniaxially at $50 \mathrm{MPa}$ in vacuum. The sintering temperature was elevated at a rate of $50^{\circ} \mathrm{C} / \mathrm{min}$ to $1200^{\circ} \mathrm{C}$. After maintaining the temperature for $10 \mathrm{~min}$, the sample was slowly cooled to $600^{\circ} \mathrm{C}$ at a rate of $5^{\circ} \mathrm{C} / \mathrm{min}$. The electric current was then stopped, the pressure was released, and the sample was cooled to room temperature.

The resulting bulk sample has high optical transmittance at wavelengths above $700 \mathrm{~nm}$ to at least $1100 \mathrm{~nm}$ (i.e., $>70 \%$ for a 1 -mm-thick sample), and a density $>99.7 \%$ of the theoretical value. As for the crystal orientation, the researchers concluded from $x$-ray diffraction measurements taken perpendicular and parallel to the direction of the pressure that there is a very significant crystal orientation on the $a, b$, and $c$ planes. The $c$ plane is aligned parallel to the pressure direction, while the $a$ and $b$ planes are aligned perpendicular to the pressure direction. Orientation indexes of the $a$ and $b$ planes are much higher than those of samples obtained with other processes.

SHIMING WU

\section{$\mathrm{LaPO}_{4}: \mathrm{Eu}^{3+}$ Nanowires Luminesce More Efficiently than Dots}

One-dimensional $\mathrm{LaPO}_{4}: \mathrm{Eu}^{3+}$ nanowires luminesce more efficiently than zero-dimensional, spherical $\mathrm{LaPO}_{4}: \mathrm{Eu}^{3+}$ nanoparticles, said Hongwei Song and co-workers from the Chinese Academy of Sciences. As reported in the March 1 issue of Optics Letters (p. 483), the researchers studied the luminescent properties of low-dimensional $\mathrm{LaPO}_{4}$ doped with rare-earth $\mathrm{Eu}$ in order to better understand the fundamental physics of this new condensed-matter system. With the technological drive toward smaller and novel devices, $\mathrm{LaPO}_{4}$ is appropriately studied as it is used in fluorescent lamps, cathode ray tubes, and plasma display panels.

The research team reported that the $\mathrm{Eu}$ atoms partially filled $f$ orbitals, which carry magnetic moments. In addition, $\mathrm{Eu}^{3+}$ ions are sensitive activators for use in the study of local symmetry. The researchers used this property of $\mathrm{Eu}^{3+}$ to study the structural differences between nanowires, nanoparticles, micron-sized powders, and micron-sized rods.

The $\mathrm{LaPO}_{4}$ :Eu nanowires, nanoparticles, micropowders, and microrods were prepared by a wet chemical method. The colloidal nanoparticles and micropowders were basic and the nanowires and microrods were acid. The diameter of the nanoparticles and nanowires ranged from $10-20 \mathrm{~nm}$, whereas the length of nanowires ranged by several hundred nanometers. The diameters of micropowders ranged over 1-2 $\mu \mathrm{m}$. The diameters and lengths of the microrods were $\sim 200 \mathrm{~nm}$ and 1-2 $\mu \mathrm{m}$, respectively. X-ray diffraction patterns revealed that the crystal structures of all of the samples belong to the monoclinic monazite type. Time-resolved emission spectra and high-resolution transmission electron micrograph images show that the $\mathrm{Eu}^{3+}$ ions occupy two sites in the nanowires as well as the microrods, but not in the nanoparticles or the micropowders. The researchers attributed this result to a degeneration of the crystal field in the micropowders. They also concluded that the observed higher radiative rate for nanowires, as compared with the nanoparticles, micropowders, and microrods, was due to the "variation of the electronicmagnetic dipole field caused by shape anisotropy."

VIVEK RANJAN

\section{Raman Measurements in Silicon Nanowires Reveal the Diameter Dependence of Phonon Confinement}

Phonon confinement in a small nanosystem leads to an asymmetric broadening of the Raman bands. Recently, P.C. Eklund from the Pennsylvania State University, G.U. Sumanasekera from the University of Louisville, and their co-workers determined the evolution of phonon confinement with wire diameter of long, crystalline Si nanowires using the Raman microprobe method.

As reported in the March 1 issue of NanoLetters (p. 409; DOI: 10.1021/nl0486259), the researchers prepared Si nanowires by pulsed $\mathrm{Nd}: Y A G$ laser vaporization of a $\mathrm{Si}$ target containing $\sim 10$ at.\% Fe. The wire growth process occurs through the vapor-liquid-solid mechanism in which a 